\title{
A Load Profile Management Integrated Power Dispatch Using a Newton-Like Particle Swarm Optimization Method
}

\author{
Caisheng Wang ${ }^{1}$ \\ Senior Member, IEEE \\ Department of Electrical and Computer Engineering \\ Wayne State University, Detroit, USA \\ cwang@wayne.edu \\ Carol J. Miller \\ Department of Civil and Environmental Engineering \\ Wayne State University \\ Detroit, USA \\ cmiller@eng.wayne.edu \\ M. Hashem Nehrir \\ Life Fellow, IEEE \\ Department of Electrical and Computer Engineering \\ Montana State University, Bozeman, MT \\ hnehrir@ece.montana.edu
}

\begin{abstract}
Load profile management (LPM) is an effective demand side management (DSM) tool for power system operation and management. This paper introduces an LPM integrated electric power dispatch algorithm to minimize the overall production cost over a given period under study by considering both fuel cost and emission factors. A Newton-like particle swarm optimization (PSO) algorithm has been developed to implement the LPM integrated optimal power dispatch. The proposed Newton-like method is embedded into the PSO algorithm to help handle equality constraints while penalty/fitness functions are used to deal with inequality constraints. In addition to illustrative example applications of the proposed Newton-like PSO technique, the optimization method has been used to realize the LPM integrated optimal power dispatch for the IEEE RTS 96 system. Simulation studies have been carried out for different scenarios with different levels of load management. The simulation results show that the LPM can help reduce generation costs and emissions. The results also verify the effectiveness of the proposed Newton-like PSO method.
\end{abstract}

Keywords -Economic/environmental dispatch; load management; Newton-like method; optimization; particle swarm optimization (PSO).

\section{INTRODUCTION}

$\mathrm{I}_{\mathrm{b}}$ $\mathrm{N}$ recent years, there has been increasing attention on airborne contaminants that contribute to climate change, terrestrial contamination, uptake of toxins in the food chain, asthma and other related health issues. Smog in China, for example, has produced great concern [1] and is considered one of the largest environmental threats to the public health of the country. The electric power industry is a major source of air pollutant emissions. In the US, about $40 \%$ of all $\mathrm{CO}_{2}$

1 Corresponding Author

This project is supported by the Great Lakes Protection Fund under Award \# GLPF-881 and partially by National Science Foundation under Award \# ECS- 1202133 .

\author{
John W. Sheppard \\ Fellow, IEEE \\ Department of Computer Science \\ Montana State University \\ Bozeman, MT \\ john.sheppard@cs.montana.edu \\ Shawn P. McElmurry \\ Department of Civil and Environmental Engineering \\ Wayne State University \\ Detroit, USA \\ s.mcelmurry@wayne.edu
}

emissions are attributed to electricity generation [2]; in 2012, 2,039 million metric ton of $\mathrm{CO}_{2}$ were generated in the US electric power sector alone [2].

Various approaches have been proposed to reduce emissions due to electricity generation. These include air pollution control devices (e.g. scrubbers for thermal power plants), fuel switching, generation from renewable sources, etc. Emission sensitive power dispatch is an important method for reducing emissions due to electric power generation. In the last three decades, a number of environmental/economic dispatch (EED) algorithms have been developed [3]-[17]. Similar to an economic dispatch problem, emission dispatch algorithms were formulated by either establishing an alternative objective function of emission or incorporating the emission cost into the generation cost models [3]-[5]. A good summary of algorithms for EED since 1970 was given in [7], where emission modeling issues, objectives and strategies formulation, constraints formulation, fuel switching, and different application scenarios with various time scales were reviewed. EED algorithms started as a single objective problem [3]-[5] and then evolved to a multi-objective optimization task [6], [7], [10], [15], [17].

Load/demand management (also called demand response or demand side management) techniques have been considered as an important tool to improve voltage profile, system efficiency and stability, to match stochastic output power of renewable sources and manage electric vehicles [18]-[29] [54] [56], and to reduce emissions [30], [31]. Load profile management (LPM), one of the load management methods, manages the load profile over a given period of study (e.g., a day) while total electricity consumption (energy) remains constant. LPM shows great potential in reducing emissions from electric power generation [30], [31].

Significant research attention has been focused on the 
development of efficient optimization techniques for EED problems. In addition to the classic Newton-Raphson and Lagrange multiplier methods [3]-[7], various heuristic/evolutionary based algorithms such as genetic algorithm (GA), particle swarm optimization (PSO) and differential evolution (DE) have been proposed for solving EED problems [9]-[10], [12], [14], [16]. Other optimization techniques such as abductive reasoning network (ARN) [8], simulated annealing (SA) [11], gravitational search algorithm (GSA) [15] and fuzzy satisfaction-maximizing decision approach [17] have also been developed for real-time dispatch and combined economic/emission optimization. Three evolutionary algorithms were investigated and compared for the dispatch of generation and load while only the generation cost was considered in the optimization model [53]. Recently, stochastic wind power was incorporated into EED for further reducing emissions [13], [32], [33]. Emission limitations have been considered for optimal generation dispatch [55]. However, little work has been done on incorporating load management for EED [30].

In this paper, a Newton-like PSO method is proposed for solving load profile management integrated environmental/economic dispatch (LPMIEED) problems to reduce emissions and cost. The remainder of the paper is organized as follows: The optimization problem formulation is discussed in Section II. The Newton-like PSO algorithm development and illustrative examples of the algorithm are given in Section III. Simulation studies using the IEEE RTS 96 system and the resulting power dispatch solutions are discussed in Section IV. Section V concludes the paper.

\section{PROBLEM ForMULATION}

Normally, EED algorithms are used to dispatch power among different generators to meet the total load demand in an economically/environmentally optimized way. Load management gives us another set of variables to carry out optimization. Though DSM methods may reduce the total electric energy consumption as a result of energy efficiency improvement, it is assumed in this paper that the load management will not change the total electricity consumption in a given period of time (e.g., a day). In other words, the LPM discussed in this paper will allow customer loads to be increased or reduced within certain time periods while the total energy consumption for the whole time period of study remains constant. In the following, the optimization problems of LPM integrated economic dispatch, emission dispatch, and EED will be considered.

\subsection{LPM Integrated Economic Dispatch}

The total generation cost, including all generators and the entire duration of analysis, is:

$$
F_{C}=\sum_{t=1}^{N T} \sum_{i=1}^{N G} F_{C, i}(t)
$$

where $N T$ is the total time intervals in the period of study. For example, $N T$ can be 24 for 24 one-hour intervals in a day. $N G$ is the total number of generators in the system. $F_{C, i}$ is the generation cost (\$) of generator $i$ in a unit time (e.g., one hour). Without considering the valve-point effect (i.e., a ripple-like effect in the heat rate curve during the value opening process of a multi-valve turbine) [11], a generator cost model can be expressed as a second order polynomial:

$$
F_{C, i}=C_{i}\left(k_{i, 0}+k_{i, 1} P_{G, i}+k_{i, 2} P_{G, i}^{2}\right)
$$

where $P_{G, i}(\mathrm{MW})$ and $C_{i}(\$ / \mathrm{MMBTU})$ are the output power and the fuel cost of generator $i$, respectively. $k_{i, j},(j=1,2,3)$ is the corresponding heat rate coefficient of generator $i$.

The total load demand in the system at time $t$ is:

$$
L(t)=\sum_{i=1}^{N L} L_{i}(t)
$$

where $N L$ is the total number of loads in the system. It is assumed that the overall system load can be controlled to a certain extent such that the new load is $[1-\mu(t)] L(t) . \mu(t)$ is the load controlling factor. If $\mu(t)$ is positive (negative), it means that the load at time interval $t$ will be reduced (increased) to $[1-\mu(t)]$ of its original value. As aforementioned, the total electricity consumption in the entire duration of study is assumed to be unaffected by load management, which gives the following equality constraint:

$$
\sum_{t=1}^{N T} \mu(t) L(t)=0
$$

There are other constraints, such as power balance, generation limits, etc., that also need to be satisfied at each time interval $t$.

$$
\begin{aligned}
& H\left(\boldsymbol{P}_{G}\right)=\sum_{i=1}^{N G} P_{G, i}(t)-[1-\mu(t)] L(t) \\
& \quad+\boldsymbol{P}_{G}^{T} \boldsymbol{B}_{\mathbf{2}} \boldsymbol{P}_{\boldsymbol{G}}+\boldsymbol{B}_{\mathbf{1}} \boldsymbol{P}_{\boldsymbol{G}}+B_{0}=0 \\
& P_{G, i, \min } \leq P_{G, i}(t) \leq P_{G, i, \max } \\
& -\mu_{\text {max }} \leq \mu(t) \leq \mu_{\max }
\end{aligned}
$$

The term $\boldsymbol{P}_{\boldsymbol{G}}^{\boldsymbol{T}} \boldsymbol{B}_{\mathbf{2}} \boldsymbol{P}_{\boldsymbol{G}}+\boldsymbol{B}_{\mathbf{1}} \boldsymbol{P}_{\boldsymbol{G}}+B_{0}$ in (5) is used to model the system power loss, where $\boldsymbol{P}_{\boldsymbol{G}}$ is the generator output power vector and $\boldsymbol{P}_{\boldsymbol{G}}=\left[\begin{array}{lll}P_{G, 1} & \cdots & P_{G, N G}\end{array}\right]^{\boldsymbol{T}} . \boldsymbol{B}_{\mathbf{2}}, \boldsymbol{B}_{\mathbf{1}}$ and $B_{0}$ are the coefficients for calculating power loss; $\boldsymbol{B}_{2}$ is a $N G \times N G$ matrix, $\boldsymbol{B}_{\mathbf{1}}$ is an $1 \times N G$ vector and $B_{0}$ is a scalar constant [34]. In (7), $0 \leq \mu_{\max } \leq 1$.

An LPM integrated economic dispatch problem can then be formulated as

$$
\begin{aligned}
& \underbrace{\operatorname{Min}}_{P_{G}, \mu} F_{C} \\
& \begin{array}{l}
\text { Subject to } \\
\text { the constraints in (4)-(7) }
\end{array}
\end{aligned}
$$

It should be noted that in a real power system there are 
other constraints (e.g., voltage regulation, security constraints) which are not included in this study. The generation unit stat-up costs and other unit commitment constraints are not considered in this paper as well.

\subsection{LPM Integrated Emission Dispatch}

Modeling of generator emissions is one of the central issues for EED. The $\mathrm{CO}_{2}$ emission is considered in this paper. The relationship between $\mathrm{CO}_{2}$ emission and generator output power can be modeled as:

$F_{E, i}=E_{i} \times\left(k_{i, 0}+k_{i, 1} P_{G, i}+k_{i, 2} P_{G, i}^{2}\right)$

where $F_{E, i}$ (ton) is the amount of $\mathrm{CO}_{2}$ emission of generator $i$, and $E_{i}$ (ton/MMBtu) is the emission factor of generator $i$.

The total emission (ton) during the entire period of study is:

$$
F_{E}=\sum_{t=1}^{N T} \sum_{i=1}^{N G} F_{E, i}(t)
$$

An LPM integrated emission dispatch problem can then be written as

$$
\begin{aligned}
& \underbrace{\operatorname{Min}}_{P_{G}, \mu} F_{E} \\
& \text { Subject to }
\end{aligned}
$$

the constraints in (4)-(7)

\subsection{LPM Integrated Economic/Environmental Dispatch (LPMIEED)}

In general, the LMPIEED is a multi-objective optimization problem. Nevertheless, it is typical to use a weight factor to combine the objective functions in (1) and (10) and to convert the problem into a single-objective optimization problem [10]. If the cost of emission can be quantified, then it also turns into a single-objective problem as:

$$
\underbrace{\operatorname{Min}}_{P_{G}, \mu}\left\{F=F_{C}+\lambda F_{E}\right\}
$$

Subject to

the constraints in (4)-(7)

where $\lambda$ (\$/ton) is the emission cost factor.

\section{Newton-Like Particle Swarm Optimization}

PSO is one of many heuristic algorithms; it shares many similarities with evolutionary computation techniques such as genetic algorithms (GA). However, in some cases it is easier and faster to implement PSO than GA due to inherent difficulties in data representation that evolutionary operators such as crossover and mutation utilize. Since it was introduced in 1995, PSO has been used in many areas including nonlinear optimization, control and artificial intelligence [35].

PSO was originally developed for solving unconstrained nonlinear optimization problems [36]. Since real optimization problems are normally constrained, several variants of PSO capable of handling constraints have been proposed [35], [37]-
[46]. The most common way of handling constraints for PSO is to convert the constrained problem into a non-constrained problem by assigning certain penalty factors into the original objective function [44]. Ranking schemes were proposed in [37] and [45] to handle constraints so that leaders with better performance were chosen to set the directions for the rest in the swarm. $\mathrm{Xu}$ and Eberhart presented a feasibility-checking method to handle constraints for PSO [38], [39]. Luo et al. employed a method of reduced space transformation to transform the problem into one without equality constraints [46]. Liang et al. proposed a dynamic multi-swarm approach to handle constraints in each subpopulation [43]. Nevertheless, the complexity of constraints that exist in real world problems remains one of the most difficult challenges in using PSO. It is particularly challenging in those situations with highly constrained search spaces. The optimums might be achieved with active constraints, or the equality constraints must be satisfied with great accuracy. Contrary to its fast spread to applications in many different areas, research on finding a general method of handling constraints for PSO has moved at a relatively slow pace.

In this paper, a Newton-like method is proposed to handle constraints for PSO based on the extension of the preliminary work reported in [47]. The proposed mechanism offers the freedom for particles to search spaces and also gives them directions to update their positions. This new method is verified by solving benchmark problems reported in literature [37], [48] and is used for solving the LPMIEED problem discussed in Section II.

\subsection{Fundamentals of PSO Algorithm}

PSO is a population based stochastic optimization technique, inspired by social swarming behavior such as bird flocking or fish schooling. Each particle in the population represents a candidate solution. All the particles start with randomly initialized positions and then "fly" throughout the search space to find the best possible solution to the problem. During the process, the particles communicate with each other and promulgate the best local solutions/positions that each of them has achieved. Then, based on the global and local information obtained, each particle updates its position towards a desired global optimum.

The basic elements of a global version of PSO algorithm are summarized below.

- Particle, $X_{j}(t)$ : Each particle is a candidate solution vector containing $n$ optimization variables. $X_{j}(t)$ is the $j$ th particle at time $t$, and it can be described as:

$X_{j}(t)=\left[\begin{array}{llll}x_{j, 1}(t), & x_{j, 2}(t), & \cdots & x_{j, n}(t)\end{array}\right]^{T}$

This particle vector is said to describe the particle's "position" within the solution space.

- Population, $P о р и(t)$ : It is a set consisting of $m$ particles at time $t$, i.e., $\operatorname{Popu}(t)=\left[\begin{array}{llll}X_{1}(t), & X_{2}(t), & \cdots & X_{m}(t)\end{array}\right]^{T}$.

- Particle Velocity, $V_{j}(t)$ : The velocity of the $j$ th particle at time $t$ in the $n$-dimensional search space can be represented as: $V_{j}(t)=\left[\begin{array}{llll}v_{j, 1}(t), & v_{j, 2}(t), & \cdots & v_{j, n}(t)\end{array}\right]^{T}$

The velocity of the particle indicates its relative change within the solution space with respect to its current position 
vector. For each time increment, a particle's velocity represents the time rate of change of the particle's solution vector.

- Personal best, $X_{j, \text { pbest }}(t)$ : It is the best position that the $j$ th particle has achieved so far at time $t$. Each particle saves its best position throughout the whole searching procedure.

- Global best, $X_{\text {gbest }}(t)$ : It is the best position (or solution) that has been achieved so far among all the particles. The information of global best is known to each and every particle in the population through communication among particles.

The original PSO algorithm is implemented as follows:

- Initialization: At the starting point $t=0$, all particles are initialized with a randomly assigned position and velocity value.

- Velocity updating: During each iteration cycle, the particle velocity is updated based on the following formula:

$$
\begin{aligned}
V_{j}(t)= & w(t) V_{j}(t-1)+c_{1} r_{1}\left[X_{j, p b e s t}(t-1)-X_{j}(t-1)\right] \\
& +c_{2} r_{2}\left[X_{\text {gbest }}(t-1)-X_{j}(t-1)\right]
\end{aligned}
$$

where $w(t)$ is the inertia weighting factor, $c_{1}$ and $c_{2}$ are two positive constants, and $r_{1}$ and $r_{2}$ are uniform random numbers in the range of $[0,1]$.

- Position updates: After updating its velocity, each particle changes its position (or solution) according to the following simple formula:

$$
X_{j}(t)=X_{j}(t-1)+V_{j}(t)
$$

\subsection{Newton's Method for Solving Nonlinear Equations}

Newton's method, also called Newton-Raphson's method, is one of the most commonly used techniques for solving nonlinear equations [49]. The iterative procedure can be described as

$$
X(t+1)=X(t)-J^{-1}(t) F(X(t))
$$

Where $\boldsymbol{X}(t)$ is the $N$-dimensional variable vector at the $t$ th step, $\boldsymbol{F}$ is the $\mathrm{N}$-dimensional function vector, and $\boldsymbol{J}$ is the Jacobian matrix.

For a set of underdetermined nonlinear equations (i.e., the number of variables is larger than the number of consistent equations), a Newton-like method can be used to find a solution with minimum norm by using the pseudoinverse [49]. A similar iterative formula to (12) can be used as follows:

$$
X(t+1)=X(t)-\operatorname{pinv}(J(t)) F(X(t))
$$

Where pinv represents the pseudoinverse operation. In this case, $J(t)$ is an $M \times N$ matrix, i.e. not a square matrix, i.e. $M \neq N$. $M$ is the number of equations, $N$ is the number of variables, and $M<N$.

Newton's method is fast and it can achieve quadratic convergence. It is a common application to combine Newton's method with other methods in a hybrid format to achieve numerical global convergence [49].

\subsection{Newton-Like Method Based PSO}

In general, the group of equality constraints such as those given in (5) is a set of underdetermined equations, which should have a set of solutions. Otherwise, there is either no solution to the optimization problem or there is no need to carry out the optimization study if only the equality constraint set itself can determine the solution of the problem.
The idea of using a Newton-like method to solve a set of underdetermined nonlinear equations can be applied to guide particles in a population to change their velocities and positions to meet the equality constraints. Fig 1 shows the flow chart of the proposed Newton-like PSO algorithm. The implementation pseudo code of the new PSO is given in Table 1.

TABLE 1

NewTON-LIKE METHOD BASED PSO
Random Initialization
Do
For each particle
PSO Velocity Update $\rightarrow V_{j}(t)$
PSO Position Update $\rightarrow X_{j}(t)$
Find the Jacobian matrix $J_{j}(t)$
$d X_{j}(t)=-\operatorname{pinv}\left(J_{j}(t)\right) H\left(X_{j}(t)\right)$
$X_{j}(t+1)=X_{j}(t)+d X_{j}(t)$
Evaluate objective function value
Update individual best

\section{End For}
Update global best
While (stop criteria not met)

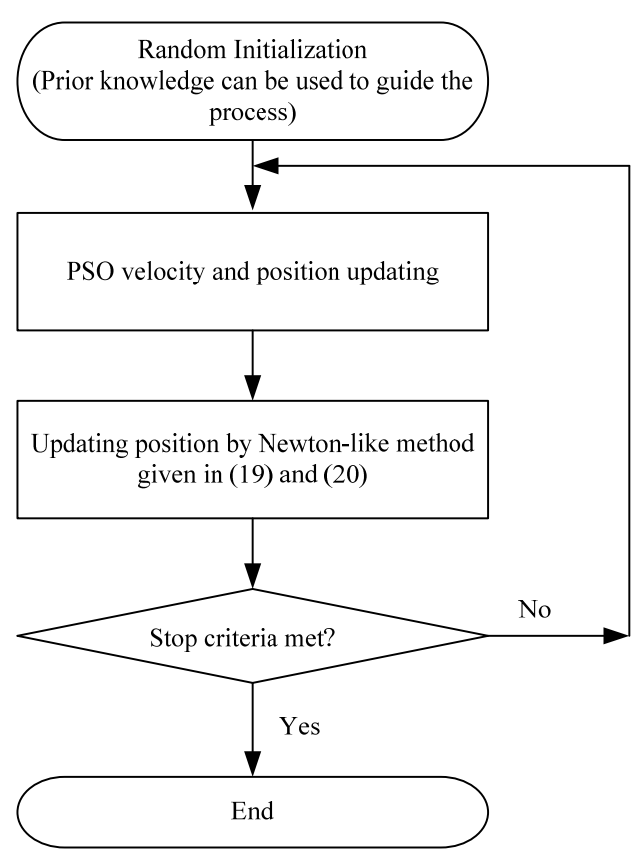

Fig. 1. Flow chart of the proposed Newton-like PSO algorithm.

In (19), $d X_{j}(t)$ is the incremental change of particle $X_{j}$ at time $t, H$ is the equality constraint vector, and $J_{j}$ is the Jacobian matrix of particle $X_{j}$.

Fig. 2 shows a graphic illustration of using the Newton-like method to handle equality constraints for PSO. Suppose that there are several particles searching in a 3-D space for an optimal solution with some given objective function. In this example, the objective function is subjected to a sphere equality constraint surface, i.e., $x_{1}^{2}+x_{2}^{2}+x_{3}^{2}=1$.

As shown in the figure, particles $X_{j}$ and $X_{k}$ are flying in the search space and updating their positions based on their 
current velocities $\left(V_{j}\right.$ and $\left.V_{k}\right)$, which are merely determined by (15). In the original PSO algorithm, $X_{j}$ and $X_{k}$ do not know explicitly where the constraint surface is and how to satisfy the constraint. With the help from the Newton-like method, an additional position adjustment, determined by (19), is added to each particle to push it toward the constraint surface. As shown in Fig. $2, d X_{j}$, for example, is added to change the next position of $X_{j}$ so that $X_{j}$ will be pulled toward the sphere surface to satisfy the equality constraint.

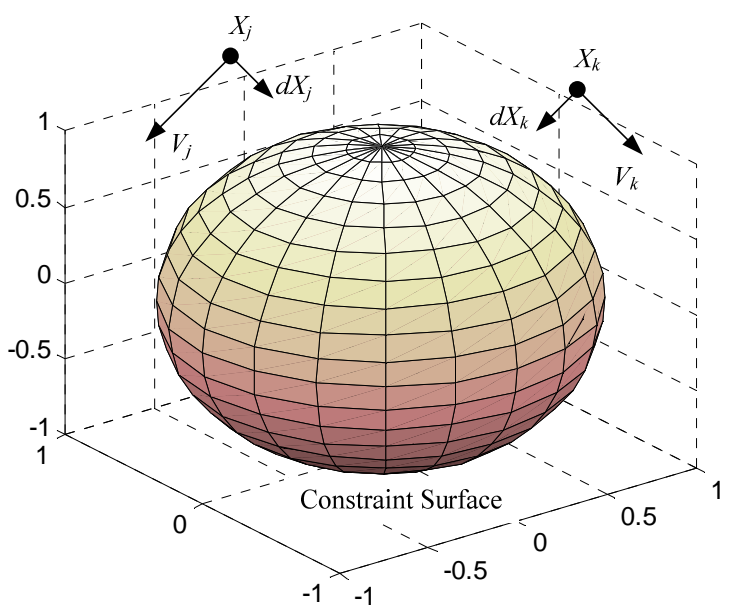

Fig. 2. Graphic illustration of handling equality constraints for PSO using Newton's method.

\subsection{Illustrative Examples of the Newton-Like Method Based PSO}

The proposed Newton-like method based PSO has been tested on the benchmark problems reported in the literature [37], [48]. Three of those benchmark problems have been chosen as illustrative examples to show the effectiveness of the proposed method. The details of the test problems are given in the Appendix. For each of the three benchmark problems, the proposed PSO program was run thirty times. The test results are summarized in Table 2, which includes the best, mean, median and worst results of the thirty runs. The standard deviation (STD), feasible rate (FR) and success rate (SR) are also given in the table. Feasible rate is defined as the number of feasible runs (where a feasible solution is found) over the total number of runs. Similarly, success rate is defined as the ratio of the number of successful runs over the total number of runs [48]. A solution is considered feasible when all the constraints are met within a tolerance of 0.001 . A successful run is a feasible run that also satisfies the following condition:

$$
\left|\frac{f(X)-f\left(X^{*}\right)}{f\left(X^{*}\right)}\right|<0.1 \%
$$

where $f(X)$ is the objective function value obtained and $f\left(X^{*}\right)$ is the best known value as reported in [48].

The test results given in Table 2 show that the proposed Newton-like method based PSO algorithm found the global optimal solutions with $100 \%$ feasible rate and success rate for those test problems. For the purpose of comparison, the results of using a regular PSO method are also given in Table 2. The regular PSO uses a penalty function to handle equality constraints [44]. The regular PSO algorithm has the same parameters as the proposed method except the steps of (19) and (20) given in Table 1. The comparative results show the effectiveness of the proposed method and indicate the incapability of the original PSO method in handling equality constraints.

TABLE 2

TEST RESUlTS OF THE PSO METHODS

\begin{tabular}{cccc}
\hline \hline \multicolumn{4}{c}{ The Proposed PSO Method } \\
\hline Problem & $g 01$ & $g 02$ & $g 03$ \\
\hline Best & -47.7210036744 & 961.715171 & 193.73785137869 \\
Worst & -47.5590286644 & 961.715546 & 193.79098756201 \\
Mean & -47.6433222857 & 961.71521 & 193.78661571604 \\
Median & -47.6483333260 & 961.715183 & 193.78819883169 \\
STD & 0.041543549703 & $7.44336 \mathrm{E}-5$ & 0.0092241458052 \\
\hline FR & $100 \%$ & $100 \%$ & $100 \%$ \\
SR & $100 \%$ & $100 \%$ & $100 \%$ \\
\hline \multicolumn{5}{c}{ Regular PSO Method } \\
\hline FR & $76.67 \%$ & $93.33 \%$ & $0 \%$ \\
SR & $0 \%$ & $33.33 \%$ & $0 \%$ \\
\hline
\end{tabular}

The proposed method has also been used to solve the LPMIEED problem discussed in Section 2. The details of the application are given in the following section.

\section{Simulation System AND SimUlation Results}

The load profile management integrated environmental/economic dispatch discussed in Section II has been implemented using the proposed Newton-like PSO method. The algorithm was tested on the 73-bus IEEE RTS 96 test system [50]. Shown in Fig. 3, the IEEE RTS 96 system represents a relatively large and complex power system, as it has 73 buses (51 load buses) and 99 generators, and a total of 8550 MW load and 10215 MW generation capacities. The generation capacity originally consists of $900 \mathrm{MW}$ hydroelectric, $2400 \mathrm{MW}$ nuclear, $3822 \mathrm{MW}$ coal, $2853 \mathrm{MW}$ petroleum, and $240 \mathrm{MW}$ petroleum combustion turbine generating units. In order to represent the increase in the use of natural gas units in recent years, $1080 \mathrm{MW}$ petroleum units in the test system were replaced with $1080 \mathrm{MW}$ natural gas units in the numerical simulation studies presented in this paper. The costs and emissions factors for hydro-electric and nuclear were set to zero so that these generators always provide base load during the analyses performed. In other words, these generators (in a total of 24) will not participate in the environmental/economic dispatch. In addition, there are 3 synchronous condensers which are solely for reactive power support. The remaining 72 generators can be grouped into three categories based on their fuel types: Coal, natural gas and oil, as given in Table 3 . These 72 generators together with the load profile management will be optimized for each hour interval in a day. Hence, the total number of decision variables of the LPMIEED problem is 1,752 (i.e., $24 \times 72+24=1,752$ ). The decision variable vector can be represented as

$$
x=\left[\begin{array}{lllll}
P_{G, 1}(1) & \cdots & P_{G, 72}(24), \mu(1) & \cdots & \mu(24)
\end{array}\right]^{T} .
$$


TABLE 3

GENERATOR TYPES IN THE REVISED IEEE RTS 96 SYSTEM

\begin{tabular}{ll}
\hline \hline Fuel Type & Generator Numbers \\
\hline \multirow{2}{*}{ Coal } & $33,4,7,8,21,22,31,32,33,36,37,40,41$, \\
Natural Gas & $54,55,64,65,66,69,70,73,74,87,88,97,98,99$ \\
& $9,10,11,16,17,18,19,20,42,43,44,49,50,51,52,53$, \\
Oil & $75,76,77,82,83,84,85,86$ \\
& $1,2,5,6,12,13,14,34,35,38,39,45,46,47$, \\
Hydro and Nuclear & $67,68,71,72,78,79,80$ \\
& $15^{*}, 23,24,25,26,27,28,29,30,48^{*}, 56,57,58,59$, \\
Unit Type Code & $60,61,62,63,81^{*}, 89,90,91,92,93,94,95,96$ \\
\hline U12 & $16,17,18,19,20,49,50,51,52,53,82,83,84,85,86$ \\
U20 & $1,2,5,6,34,35,38,39,67,68,71,72$ \\
& $15^{*}, 25,26,27,28,29,30,48 *, 58,59,60,61$, \\
U50 & $62,63,81 *, 91,92,93,94,95,96$ \\
& $3,4,7,8,36,37,40,41,69,70,73,74$ \\
U16 & $9,10,11,42,43,44,75,76,77$ \\
U150 & $21,22,31,32,54,55,64,65,87,88,97,98$ \\
U197 & $12,13,14,45,46,47,78,79,80$ \\
U350 & $33,66,99$ \\
U400 & $23,24,56,57,89,90$ \\
\hline$* \cdot$ Synchronous Condensers
\end{tabular}

*: Synchronous Condensers

\subsection{Fuel Costs, Emission Factors and Load Profile}

Table 4 shows the fuel costs and emission factors of generators based on their fuel types. The data are taken from [51] and [52]. The heat rate parameters (i.e., $k_{i, 0}, k_{i, 1}$ and $k_{i, 2}$ ) of different unit types are taken from [50], given in Table 5.

Table 6 lists the hourly load profile in a day, which is similar to the one used in [16] but was scaled up based on the average load demand of the IEEE RTS 96 system.

TABLE 4

FUEL COSTS AND EMISSION FACTORS

\begin{tabular}{lccc}
\hline & Coal & Natural Gas & Oil \\
\hline $\begin{array}{l}\text { Fuel Cost } \\
(\$ / \text { MMBtu })\end{array}$ & 2.05 & 6.30 & 9.05 \\
\hline
\end{tabular}

\begin{tabular}{lccc}
\hline \hline $\begin{array}{l}\text { Emission Factor } \\
\text { (Metric ton/MMBtu) }\end{array}$ & 0.0953 & 0.0535 & 0.0735 \\
\hline
\end{tabular}

TABLE 5 HEAT RATE PARAMETERS

\begin{tabular}{llll}
\hline \hline & \multicolumn{4}{l}{$k_{i, 2}$} & $k_{i, 1}$ & $k_{i, 0}$ \\
\hline U12 & 0.1582 & 8.6806 & 16.8204 \\
U20 & 0.5471 & -7.2019 & 215.1852 \\
U50 & 0 & 0 & 0 \\
U76 & 0.0309 & 7.8650 & 134.1222 \\
U100 & 0.0119 & 7.5097 & 129.7522 \\
U155 & 0.0056 & 7.5464 & 184.3804 \\
U197 & 0.0050 & 7.6571 & 189.6178 \\
U350 & 0.0033 & 7.4330 & 323.5432 \\
U400 & 0.0010 & 8.5880 & 406.8978 \\
\hline
\end{tabular}

TABLE 6

HOURLY LOAD PROFILE

\begin{tabular}{cccc}
\multicolumn{4}{c}{ HOURLY LOAD PROFILE } \\
\hline \hline Time $(\mathrm{h})$ & Load $(\mathrm{MW})$ & Time $(\mathrm{h})$ & Load $(\mathrm{MW})$ \\
\hline 1 & 6583.5 & 13 & 9234.0 \\
2 & 6348.4 & 14 & 9747.0 \\
3 & 6305.6 & 15 & 9661.5 \\
4 & 6455.3 & 16 & 9533.3 \\
5 & 6583.5 & 17 & 9298.1 \\
6 & 6626.3 & 18 & 9105.8 \\
7 & 7481.3 & 19 & 8699.6 \\
8 & 9191.3 & 20 & 7908.8 \\
9 & 9832.5 & 21 & 7481.3 \\
10 & 9939.4 & 22 & 6840.0 \\
11 & 9875.3 & 23 & 6412.5 \\
12 & 9725.6 & 24 & 9234.0 \\
\hline
\end{tabular}

Simulation studies have been carried out for the LPM integrated power dispatch on the IEEE RTS 96 system with the fuel cost and emission factors given in Table 4 and the load profile in Table 6 . The results of the different scenarios with different levels of load management are presented, compared and discussed in the next subsections. The proposed Newton-like method based PSO is also compared with the optimization toolbox in MATLAB.

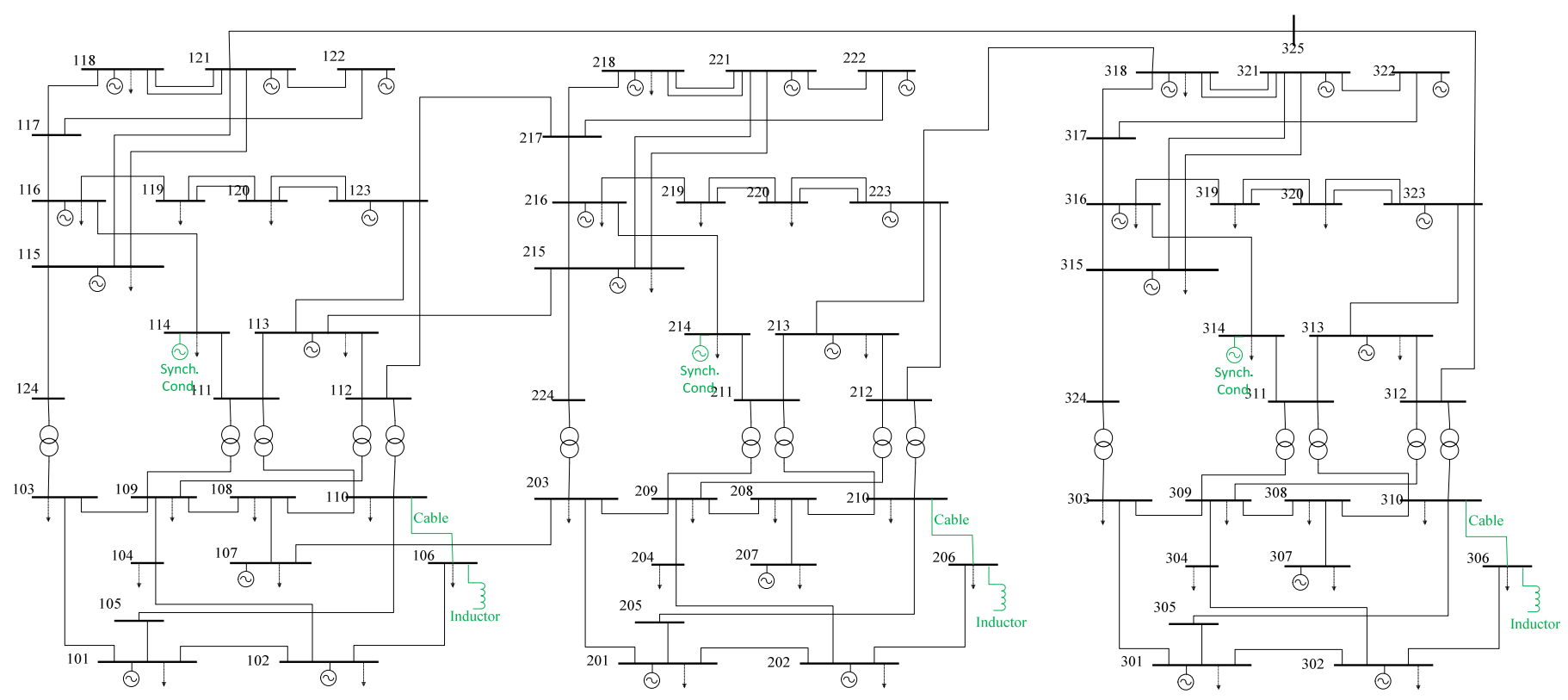

Fig. 3. IEEE RTS 96 System [50]. 


\subsection{Simulation Results}

1) Constant emission cost factor. Simulation studies are first carried out for a constant emission cost factor (see $\lambda$ in (12)). $\lambda$ is chosen as $\$ 5 /$ ton for the simulation studies. Fig. 4 shows the overall generation cost of the system at different levels of load management (i.e., different values of $\mu_{\max }$ in (7)) in the 24-hour simulation period. The case of $\mu_{\max }=0$ represents the scenario when there is no LPM. In this case, the generators are still optimally dispatched, but only on each individual hour interval, not for the whole 24 hour period. It can be noted from the figure that the generation cost is reduced since a greater portion of the load is controllable. The results also show that the generation cost savings becomes saturated when the level of controllable load exceeds approximately $30 \%\left(\mu_{\max }=0.30\right)$. Even if more loads were controllable beyond this point, no significant additional cost savings would result. This is mainly because of the load profile (in Table 6) and the cost models (in Table 4) used in the simulation studies. As shown in the figure, the LPM can reduce generation cost by nearly $10 \%$. The $\mathrm{CO}_{2}$ emission versus load management is also given in Fig. 4. It can be seen that $\mathrm{CO}_{2}$ emissions remain essentially unchanged under different levels of load management for this uniform emission cost factor case.

Fig. 5 shows the total generation profiles over the 24 hour simulation period for the case of no load management $\left(\mu_{\max }=\right.$ 0 ) and when $\mu_{\max }=0.20$. This figure demonstrates that the loads at the peak regions have been shifted to the valley zones to make the load more evenly distributed in order to reduce the overall generation cost.

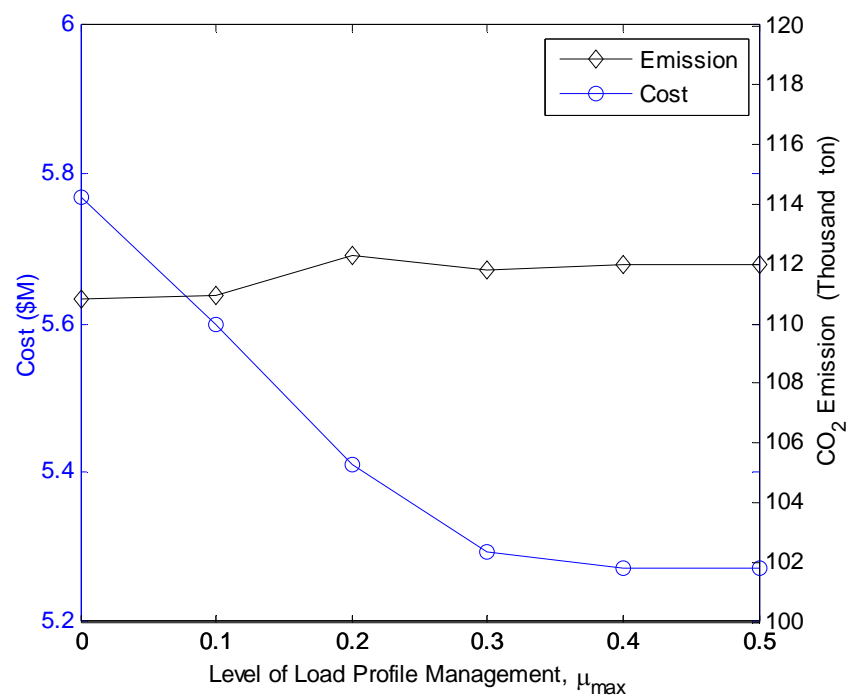

Fig. 4. Cost and emission versus different load management levels with a uniform emission cost factor $\lambda=\$ 5 /$ ton.

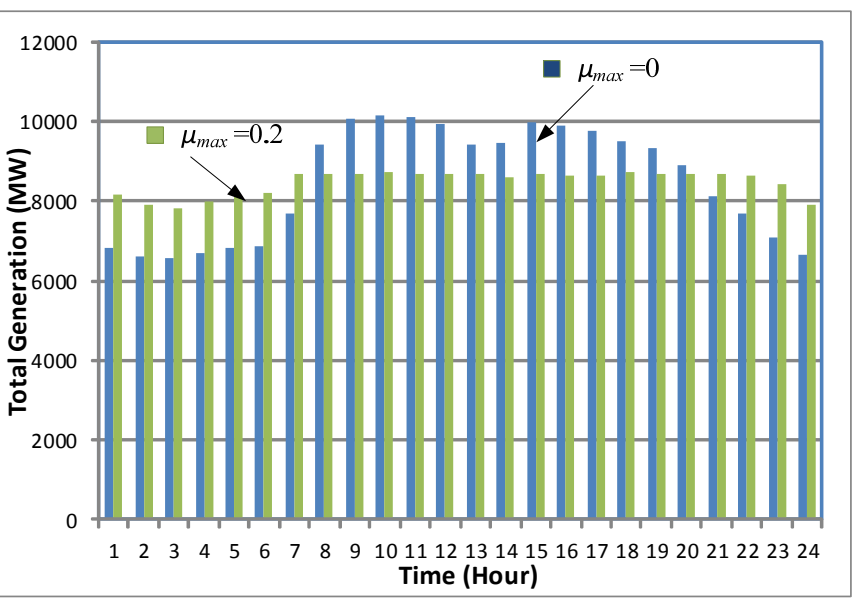

Fig. 5. The overall generation profiles of LPMIEED for the two scenarios of $\mu_{\max }=0$ and $\mu_{\max }=0.2$ with a uniform emission cost factor, $\lambda=\$ 5 /$ ton.

Fig. 6 shows the fuel mixture of the electricity generation over the 24 hour simulation period for the case of no load management $\left(\mu_{\max }=0\right)$ and when $\mu_{\max }=0.20$. It can be seen from the figure that more electricity generation has been shifted from more expensive units (i.e., oil-fired generators) to cheaper generators (i.e., coal and natural gas).

2) Constant load management level. As discussed earlier in this section, there is no significant improvement in cost savings when $\mu_{\max }$ is over 0.30 . Hence, $\mu_{\max }$ is set at 0.3 for the rest of the simulation studies. The total $\mathrm{CO}_{2}$ emissions with different emission cost factors and the corresponding fuel costs are presented in Fig. 7. As demonstrated, the total $\mathrm{CO}_{2}$ emissions are reduced if the emission is given more weight (i.e., a higher emission cost factor) in the optimization model. Compared with the economic dispatch scheme (i.e., $\lambda=0$ ), the environmental dispatch (without considering the fuel cost) can reduce the total $\mathrm{CO}_{2}$ emission by over $10.4 \%$, which is equivalent to a reduction of 11,690 metric tons in a day or a reduction of about 4.3 million metric tons in a year.

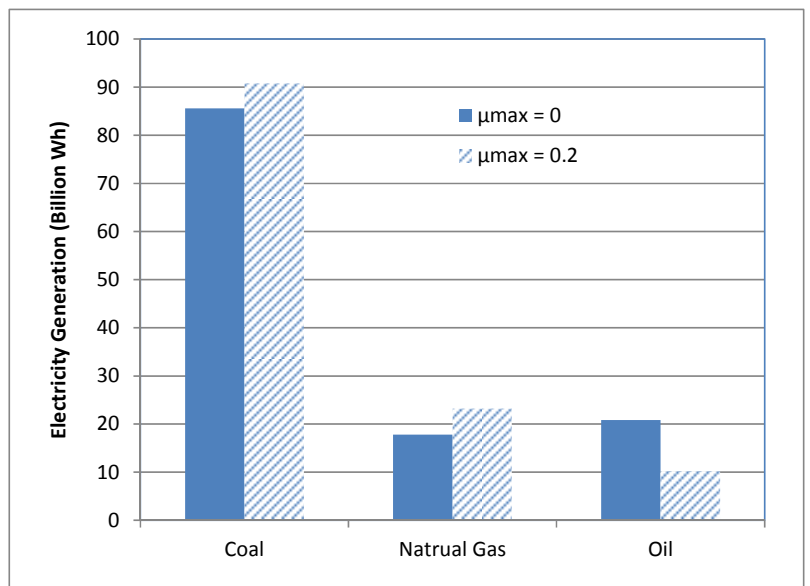

Fig. 6. The fuel mixture of LPMIEED for the two scenarios of $\mu_{\max }=0$, and $\mu_{\max }=0.2$ with an emission cost factor $\lambda=\$ 5 /$ ton. 


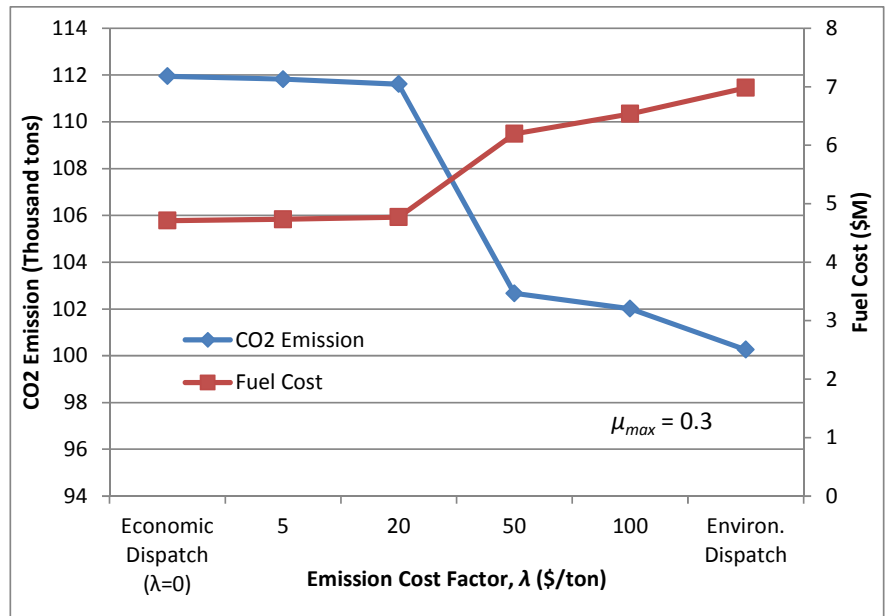

Fig. 7. $\mathrm{CO}_{2}$ emission and fuel cost versus different emission cost factors with $\mu_{\max }=0.3$.

Fig. 8 shows the fuel mixture of the economic dispatch and environmental dispatch. It clearly shows that the generation has been shifted from units with higher emission rates (i.e., coal power plants) to generators with lower emission rates (i.e., natural gas and oil) under the environmental dispatch scheme.

It can also be noted from Figs. 3 and 5-7 that the goals of fuel cost saving and emission reduction may not come together at the same optimal point. The LPMIEED with an objective function given in (12) can provide a Pareto front, shown in Fig. 9, based on which an optimal tradeoff can be made to balance the fuel cost and emission. Many practical factors will affect the selection of a particular operating point (in the Pareto front) for a given system. As environmental issues gain additional emphasis (e.g. emission reductions to mitigate climate change as suggested in Kyoto and Copenhagen Protocols), the decision may lean more toward emission reduction in the future.

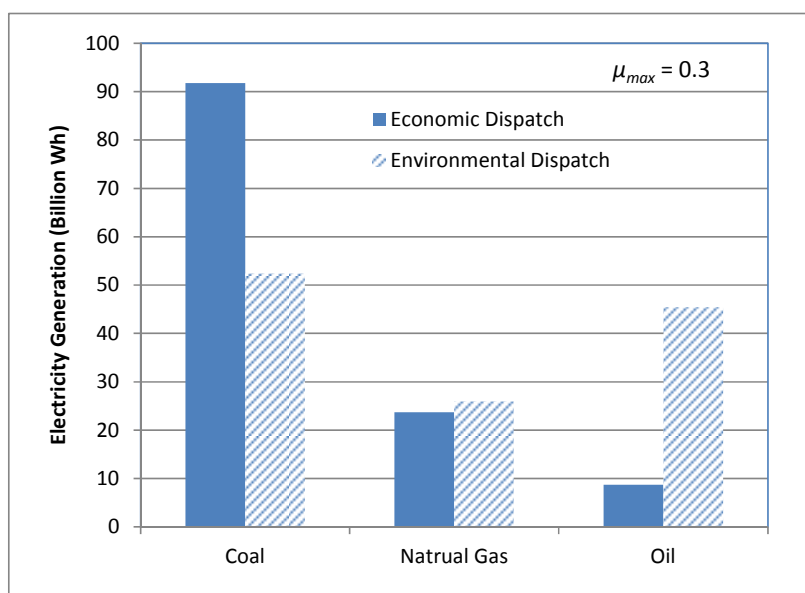

Fig. 8. The fuel mixture of LPMIEED with $\mu_{\max }=0.30$ for the two scenarios: Economic dispatch and environmental dispatch.

3) Comparison between the Newton-like PSO and the Optimization toolbox in MATLAB. The simulation studies on the IEEE RTS 96 System were carried out on a PC with an Intel(R) Core (TM) i7-3770 $3.4 \mathrm{GHz} \mathrm{CPU}$ and $8.0 \mathrm{~GB}$ memory, running on Microsoft Windows 7. All the testing and simulation programs were coded using MATLAB R2012b.

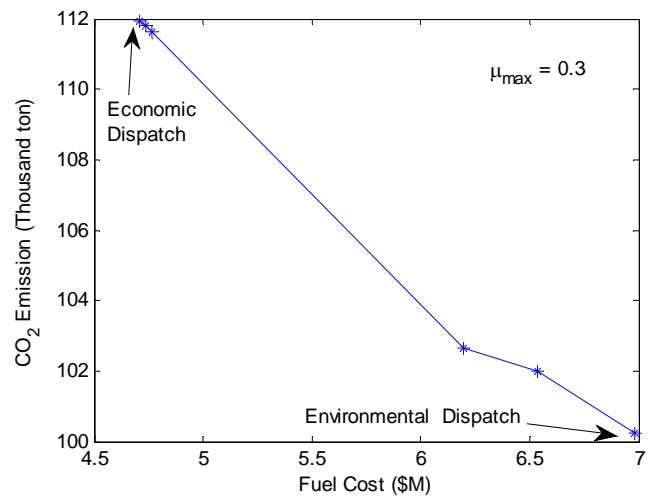

Fig. 9. The Pareto front curve of the LPMIEED with $\mu_{\max }=0.30$.

The parameter settings of the PSO programs are listed in Table 7. The inertia weighting factor, $w(t)$ in (15), is chosen as $w(t)=\alpha_{w} w(t-1)$, where $\alpha_{w}$ is the annealing factor. It took about one hour to obtain a feasible solution using the proposed PSO algorithm on the aforementioned PC.

TABLE 7

PARAMETER SETTINGS FOR THE PSO ALGORITHMS

\begin{tabular}{ll}
\hline \hline Parameter & Memo \\
\hline$\alpha_{w}=0.98$ & $w(t)=\alpha_{w} w(t-1)$, see $(15)$ \\
$c_{1}=2$ & See $(15)$ \\
$c_{2}=2$ & See $(15)$ \\
$m=8,000$ & Total number of particles \\
$N_{\text {tot }}=200$ & Maximum number of total iterations \\
$N_{L}=1000$ & Maximum number of iterations since the last update of the \\
& best solution
\end{tabular}

The fmincon function with "active-set" algorithm in the MATLAB optimization toolbox was used to implement the same LPMIEED scheme and ran on the same PC. It took about 15 hours, but the program still failed to provide a feasible solution. In other words, the fmincon optimization program stopped prematurely when the maximum iteration number exceeded the pre-set default value of 175,200 . The conventional PSO algorithm also failed to give a feasible solution. The comparison results show that the Newton-like PSO method is an effective tool in solving optimization problems with nonlinear equality constraints.

\section{CONCLUSION}

A load profile management integrated environmental/economic dispatch (LPMIEED) algorithm was formulated in this paper. A Newton-like PSO method has been developed for solving the LMPIEED problem. The algorithm was implemented in MATLAB and tested on the IEEE RTS 96 system. The simulation results show that the LPM can reduce system generation costs and emissions by about $10 \%$ for the IEEE test system. For the given load profile discussed in the paper, the simulation results also show that the effect of load profile management becomes saturated when the level of load management is over $30 \%$ for the IEEE RTS 96 system. Overall, results demonstrate that load management schemes have great potential for reducing emissions.

The proposed Newton-like method was compared with the 
optimization toolbox function in MATLAB. The illustrative examples, the simulation studies on the IEEE RTS 96 system, and the comparison results show that the proposed PSO technique is effective in finding solutions for optimization problems with equality constraints.

\section{APPENDIX}

\section{Problem g01 [48]}

$$
\begin{aligned}
& \operatorname{Min} f(X)=\sum_{i=1}^{10} x_{i}\left(c_{i}+\ln \frac{x_{i}}{\sum_{j=1}^{10} x_{j}}\right) \\
& \text { s.t. } \\
& \quad h_{1}(X)=x_{1}+2 x_{2}+3 x_{3}+x_{6}+x_{10}-2=0 \\
& h_{2}(X)=x_{4}+2 x_{5}+x_{6}+x_{7}-1=0 \\
& h_{3}(X)=x_{3}+x_{7}+x_{8}+2 x_{9}+x_{10}-1=0
\end{aligned}
$$

Where $0<x_{i} \leq 10$ for $(i=1, \ldots, 10)$, and $c_{1}=-6.089, c_{2}=-$

$17.164, c_{3}=-34.054, c_{4}=-5.914, c_{5}=-24.721, c_{6}=-14.986, c_{7}$ $=-24.1, c_{8}=-10.708, c_{9}=-26.662, c_{10}=-22.179$. The best

known solution is at $X^{*}=(0.0406684113216282$;

0.147721240492452 ;

$0.783205732104114 ; 0.00141433931889084$;

$0.485293636780388 ; 0.000693183051556082$;

$0.0274052040687766 ; 0.0179509660214818$;

$0.0373268186859717 ; 0.0968844604336845)$ with $f\left(X^{*}\right)=$ 47.7648884594915 .

\section{Problem g02 [48]}

$$
\text { Min } f(X)=1000-x_{1}^{2}-2 x_{2}^{2}-x_{3}^{2}-x_{1} x_{2}-x_{1} x_{3}
$$

s.t.

$$
\begin{aligned}
& h_{1}(X)=x_{1}^{2}+x_{2}^{2}+x_{3}^{2}-25=0 \\
& h_{2}(X)=8 x_{1}+14 x_{2}+7 x_{3}-56=0
\end{aligned}
$$

Where $0 \leq x_{i} \leq 10$ for $(i=1,2,3)$. The optimum solution is $X^{*}$ $=(3.51212812611795133 ; 0.216987510429556135$; $3.55217854929179921)$ with $f\left(X^{*}\right)=961.715022289961$

$$
\begin{aligned}
& \begin{array}{l}
\text { Problem } g 03 \\
\text { Min } f(X)=
\end{array}\left[\begin{array}{l}
18] \\
\text { Where } f_{1}\left(x_{1}\right)=\left\{\begin{array}{ll}
30 x_{1} & 0 \leq x_{1}<300 \\
31 x_{1} & 300 \leq x_{1}<400
\end{array}\right. \text { and }
\end{array}\right. \\
& \qquad f_{2}\left(x_{2}\right)=\left\{\begin{array}{cc}
28 x_{2} & 0 \leq x_{2}<100 \\
29 x_{2} & 100 \leq x_{2}<200 \\
30 x_{2} & 200 \leq x_{2}<1000
\end{array}\right. \\
& \text { S.t. } \\
& h_{1}(X)=-x_{1}+300+\frac{x_{3} x_{4}}{131.078} \cos \left(1.48477-x_{6}\right)+\frac{0.90798 x_{3}^{2}}{131.078} \cos (1.47588)=0 \\
& h_{2}(X)=-x_{2}-\frac{x_{3} x_{4}}{131.078} \cos \left(1.48477+x_{6}\right)+\frac{0.90798 x_{4}^{2}}{131.078} \cos (1.47588)=0 \\
& h_{3}(X)=-x_{5}-\frac{x_{3} x_{4}}{131.078} \sin \left(1.48477+x_{6}\right)+\frac{0.90798 x_{4}^{2}}{131.078} \sin (1.47588)=0 \\
& h_{4}(X)=200-\frac{x_{3} x_{4}}{131.078} \sin \left(1.48477-x_{6}\right)+\frac{0.90798 x_{3}^{2}}{131.078} \sin (1.47588)=0
\end{aligned}
$$

Where $0 \leq x_{1} \leq 400,0 \leq x_{2} \leq 1000,340 \leq x_{3}, x_{4} \leq 420$, $-1000 \leq x_{5} \leq 1000$ and $0 \leq x_{6} \leq 0.5236$. The best known solution is at $X^{*}=(201.784467214523659$; 99.9999999999999005;

383.071034852773266; 420;-10.9076584514292652;

$0.0731482312084287128)$ where $f\left(X^{*}\right)=8853.53967480648$.

\section{REFERENCES}

[1] D. H. a. J. Jiang, "A Study of Smog Issues and PM2.5 Pollutant Control Strategies in China," Journal of Environmental Protection, vol. 4, no. 7, pp. 746-752, 2013.

[2] "How much of U.S. carbon dioxide emissions are associated with electricity generation?" The U.S. Energy Information Administration (EIA), August 2013. [Online]. Available: http://www.eia.gov/tools/faqs/faq.cfm?id=77\&t=3. [Accessed 30 December 2013].

[3] M.R. Gent and J.W. Lamont, "Minimum emission dispatch," IEEE Transactions on Power Apparatus and Systems, vol. PAS90, no. 6, pp. 2650-60, Nov.-Dec. 1971.

[4] J.K. Delson, "Controlled emission dispatch," IEEE Transactions on Power Apparatus and Systems, vol. PAS-93, no. 5, pp. 1359-66, Sept.Oct. 1974.

[5] J.B. Cadogan, L. Eisenberg, "Sulfur oxide emissions management for electric power systems," IEEE Transactions on Power Apparatus and Systems, vol. PAS-96, no. 2, pp. 393-401, March-April 1977.

[6] J.S. Heslin, and B.F. Hobbs, "A multiobjective production costing model for analyzing emissions dispatching and fuel switching," IEEE Transactions on Power Systems, vol. 4, no. 3, pp. 836-42, Aug. 1989.

[7] J.H. Talaq, F. El-Hawary, and M.E. El-Hawary, "A summary of environmental/economic dispatch algorithms," IEEE Transactions on Power Systems, vol. 9, no. 3, pp. 1508-16, Aug. 1994.

[8] Chao-Ming Huang and Yann-Chang Huang, "A novel approach to realtime economic emission power dispatch," IEEE Transactions on Power Systems, vol. 18, no. 1, pp. 288-94, Feb. 2003.

[9] R. Gnanadass, Narayana Prasad Padhy and K. Manivannan, "Assessment of available transfer capability for practical power systems with combined economic emission dispatch," Electric Power Systems Research, vol. 69, no. 2-3, pp. 267-76, May 2004.

[10] M. A. Abido, "Environmental/economic power dispatch using multiobjective evolutionary algorithms," IEEE Transactions on Power Systems, vol. 18, no. 4, pp. 1529-1537, Nov. 2003.

[11] M. Basu, "A simulated annealing-based goal-attainment method for economic emission load dispatch of fixed head hydrothermal power systems," International Journal of Electrical Power and Energy Systems, vol. 27, no. 2, pp. 147-153, February 2005.

[12] Chao-Lung Chiang, "Optimal economic emission dispatch of hydrothermal power systems," International Journal of Electrical Power \& Energy Systems, vol. 29, no. 6, pp. 462-9, July 2007.

[13] Xian Liu and Wilsun Xu, "Minimum emission dispatch constrained by stochastic wind power availability and cost," IEEE Transactions on Power Systems, vol. 25, no. 3, pp. 1705-1713, August 2010.

[14] A.A. Abou El Ela, M.A. Abido and S.R. Spea, "Differential evolution algorithm for emission constrained economic power dispatch problem," Electric Power Systems Research, vol. 80, no. 10, pp. 1286-92, Oct. 2010.

[15] Binod Shaw, V. Mukherjee, and S.P. Ghoshal, "A novel oppositionbased gravitational search algorithm for combined economic and emission dispatch problems of power systems," International Journal of Electrical Power and Energy Systems, 2011.

[16] Gwo-Ching Liao, "A novel evolutionary algorithm for dynamic economic dispatch with energy saving and emission reduction in power system integrated wind power," Energy, vol. 36, no. 2, pp. 1018-29, Feb. 2011.

[17] Chao-Ming Huang, Hong-Tzer Yang and Ching-Lien Huang, "Biobjective power dispatch using fuzzy satisfaction-maximizing decision approach," IEEE Transactions on Power Systems, vol. 12, no. 4, p 171521, Nov. 1997.

[18] Ali Al-Alawi and S.M Islam, "Demand side management for remote area power supply systems incorporating solar irradiance model," Renewable Energy, vol. 29, pp. 2027-2036, 2004. 
[19] Q. Binh Dam, Salman Mohagheghi and James Stoupis, "Intelligent Demand Response Scheme for Customer Side Load Management," IEEE Energy 2030, Atlanta, GA US, 17-18 November, 2008.

[20] Surekha Dudhani, A.K. Sinha and S.S. Inamdar, "Renewable energy sources for peak load demand management in India," Electrical Power and Energy Systems, vol. 28, pp. 396-400, 2006.

[21] A. Molina-Garcia, J.A. Fuentes, E. Gómez-Lázaro, A. Bonastre, J.C. Campelo, and J.J. Serrano, "Application of Wireless Sensor Network to Direct Load Control in Residential Areas," IEEE International Symposium on Industrial Electronics, pp. 1974-1979, 2007.

[22] Yusuf Gurkaynak and Alireza Khaligh, "Control and Power Management of a Grid Connected Residential Photovoltaic System with Plug-in Hybrid Electric Vehicle (PHEV) Load," 2009 Twenty-Fourth Annual IEEE Applied Power Electronics Conference and Exposition (APEC), pp. 2086-91, 2009.

[23] Warsono, D. J. King, C. S. Özveren and D.A. Bradley, "Economic Load Dispatch Optimization of Renewable Energy in Power System Using Genetic Algorithm," PowerTech 2007, pp. 531-1-6, 2008.

[24] Keinosuke Matsumoto, Yasuhito Takamuki, Naoki Mori and Masash Kitayama, "An Interactive Approach to Demand Side Management Based on Utility Functions," International Conference on Electric Utility Deregulation and Restructuring and Power Technologies, pp. 147-50, 2000.

[25] Goran Strbac, "Demand side management: Benefits and challenges," Energy Policy, vol. 36, pp. 4419-4426, 2008.

[26] Dirk Westermann and Andreas John, "Demand Matching Wind Power Generation With Wide-Area Measurement and Demand-Side Management," IEEE Transactions on Energy Conversion, vol. 22, no. 1, pp. 145-,149, March 2007.

[27] Marija Ilic, Le Xie, and Jhi-Young Joo, "Efficient coordination of wind power and price-responsive demand - Part I: theoretical foundations," IEEE Transactions on Power Systems, vol. 26, no. 4, pp. 1875-1884, November 2011

[28] B.J. Kirby and R.H. Staunton, "Technical potential for peak load management programs in New Jersey," ORNL/TM-2002-271, Oak Ridge National Laboratory, Oct. 2002.

[29] Rahmat Aazami, Kaveh Aflaki and Mahmoud Reza Haghifam "A demand response based solution for LMP management in power markets," International Journal of Electrical Power and Energy Systems, vol. 33, no. 5, pp. 1125-1132, June 2011.

[30] C. Wang, S.P. McElmurry, C.J. Miller, and J. Zhao, "An Integrated Economic/Emission/Load Profile Management Dispatch Algorithm," IEEE PES General Meeting, San Diego, CA, July 2012.

[31] Y. Wang, C. Wang, C. J. Miller, S. P. McElmurry, S. S. Miller, and M. M.Rogers, "Locational Marginal Emissions: Analysis of pollutant emission reduction through spatial management of load distribution," Applied Energy, vol. 119, no. 15, pp. 141-150, 2014.

[32] Cheng-Chien Kuo and Teng-Fa Taso "Generation dispatch under large penetration of wind power considering emission and economy," Proceedings of the 2009 International Conference on Energy and Environment Technology (ICEET 2009), pp. 679-82, 2009.

[33] X. Liu, "Emission minimisation dispatch constrained by cost and wind power," IET Generation, Transmission \& Distribution, vol. 5, no. 7, pp. 735-42, July 2011

[34] Hadi Saadat, Chapter 7, Power System Analysis, McGraw Hill, (ISBN: 0-07-284869-3), 2nd edition, July 15, 2002.

[35] X. Hu, R. Eberhart, and Y. Shi, "Recent advances in particle swarm," Proceedings of IEEE Congress on Evolutionary Computation 2004, pp. 90-97, Portland, Oregon, USA.

[36] J. Kennedy and R.C. Eberhart, "Particle Swarm Optimization," Proc., The 1995 IEEE International Conference on Neural Networks, vol. 4, pp. 1942-1948, 1995.

[37] Gregorio Toscano Pulido and Carlos A. Coello, "A Constraint-Handling Mechanism for Particle Swarm Optimization," Proceedings of the 2004 Congress on Evolutionary Computation, pp. 1396-1403, 2004.

[38] X. Hu and R. C. Eberhart "Solving constrained nonlinear optimization problems with particle swarm optimization", Proc., The Sixth World Multiconference on Systemics, Cybernetics and Informatics 2002 (SCI 2002), vol. 5, 2002, Orlando, USA.

[39] Xiaohui Hu, R.C. Eberhart and Yuhui Shi, "Engineering optimization with particle swarm," Proc., The 2003 IEEE Swarm Intelligence Symposium (SIS'03), pp. 53-57, 2003.

[40] Xiao-Feng Xie, Wen-Jun Zhang, De-Chun Bi, "Handling equality constraints by adaptive relaxing rule for swarm algorithms", Proc., The
2004 IEEE Congress on Evolutionary Computation, pp. 2012-2016, 2004

[41] C.K. Monson, K.D. Seppi, "Linear equality constraints and homomorphous mappings in PSO," Proc., The 2005 IEEE Congress on Evolutionary Computation, pp. 73-80, vol. 1, 2005, Edinburgh, Scotland.

[42] Mihaela Breaban, Madalina Ionita and Cornelius Croitoru, "A new PSO approach to constraint satisfaction," Proc., The 2007 IEEE Congress on Evolutionary Computation, pp. 1948-1954, vol. 1, 2007.

[43] J. J. Liang and P. N. Suganthan, "Dynamic multi-swarm particle swarm optimizer with a novel constraint-handling mechanism," Proc., The 2006 IEEE Congress on Evolutionary Computation, pp. 424-445, July, 2006, Vancouver, BC, Canada.

[44] K. E. Parsopoulos and M. N. Vrahatis, "Particle swam optimization method for constrained optimization problems," Proceedings of the Euro-International Symposium on Computational Intelligence, 2002.

[45] T. Ray and K. M. Liew, "A swarm with an effective information sharing mechanism for unconstrained and constrained single objective optimization problem," Proceedings of IEEE Congress on Evolutionary Computation (CEC 2001), pp. 75-80, 2001, Seoul, Korea.

[46] Luo Yiqing, Yuan Xigang and Liu Yongjian, "An improved PSO algorithm for solving non-convex NLP/MINLP problems with equality constraints," Computers and Chemical Engineering, vol. 31, pp.153$162,2007$.

[47] C. Wang, M. Hashem Nehrir, LY Wang, Feng Lin, and Chris M. Colson, "Hybrid Constraint-Handling Mechanism for Particle Swarm Optimization with Applications in Power Systems," The 2011 International Conference on Genetic and Evolutionary Methods, Las Vegas, NV, July, 2011.

[48] J. J. Liang, Thomas Philip Runarsson, Efren Mezura-Montes, Maurice Clerc, P. N. Suganthan, Carlos A. Coello and K. Deb, "Problem Definitions and Evaluation Criteria for the CEC 2006 Special Session on Constrained Real-Parameter Optimization," Technical Report, Nanyang Technological University, Singapore, Dec 2005.

[49] D. R. Kincaid and E. W. Cheney, Numerical Analysis: Mathematics of Scientific Computing, 3rd Edition. Brooks/Cole, Pacific Grove, CA, 2002.

[50] C. Grigg, P. Wong, P. Albrecht, R. Allan, M. Bhavaraju, R. Billinton, Q. Chen, C. Fong, S. Haddad, S. Kuruganty, W. Li, R. Mukerji, D. Patton, N. Rau, D. Reppen, A. Schneider, M. Shahidehpour, C. Singh, "The IEEE Reliability Test System-1996. A report prepared by the Reliability Test System Task Force of the Application of Probability Methods Subcommittee," IEEE Transactions on Power Systems, pp. 1010-1020, vol. 14, no.3, 1999

[51] Air Emissions, US Environmental Protection Agency, [Online], Available: http://www.epa.gov/cleanenergy/energy-and-you/affect/airemissions.html.

[52] M. Rogers, Y. Wang, C. Wang, S.P. McElmurry, and C.J. Miller, "Evaluation of a Rapid LMP-Based Approach for Calculating Marginal Unit Emissions," Applied Energy, vol. 111, pp. 812-820, Nov., 2013.

[53] Thaís de Fátima Araújo and Wadaed Uturbey, "Performance assessment of PSO, DE and hybrid PSO-DE algorithms when applied to the dispatch of generation and demand," International Journal of Electrical Power and Energy Systems, vol. 47, pp. 205-217, 2013.

[54] M.A. López, S. de la Torre, S. Martín, and J.A. Aguado, "Demand-side management in smart grid operation considering electric vehicles load shifting and vehicle-to-grid support," International Journal of Electrical Power and Energy Systems, vol. 64, pp. 689-698, 2015.

[55] A.M. Elaiw, X. Xia, and A.M. Shehata, "Application of model predictive control to optimal dynamic dispatch of generation with emission limitations," Electric Power Systems Research, vol. 84, pp. 3144, 2012.

[56] Federico Milano, and Olivier Hersent, "Optimal Load Management with Inclusion of Electric Vehicles and Distributed Energy Resources," IEEE Transactions on Smart Grid, vol. 5, no. 2, 2014. 